
2 Research Square
Preprints are preliminary reports that have not undergone peer review.
They should not be considered conclusive, used to inform clinical practice, or referenced by the media as validated information.

\title{
Pros and Cons of Aspirin for the Primary Prevention of Cardiovascular Events: A Secondary Study of Trial Sequential Analysis
}

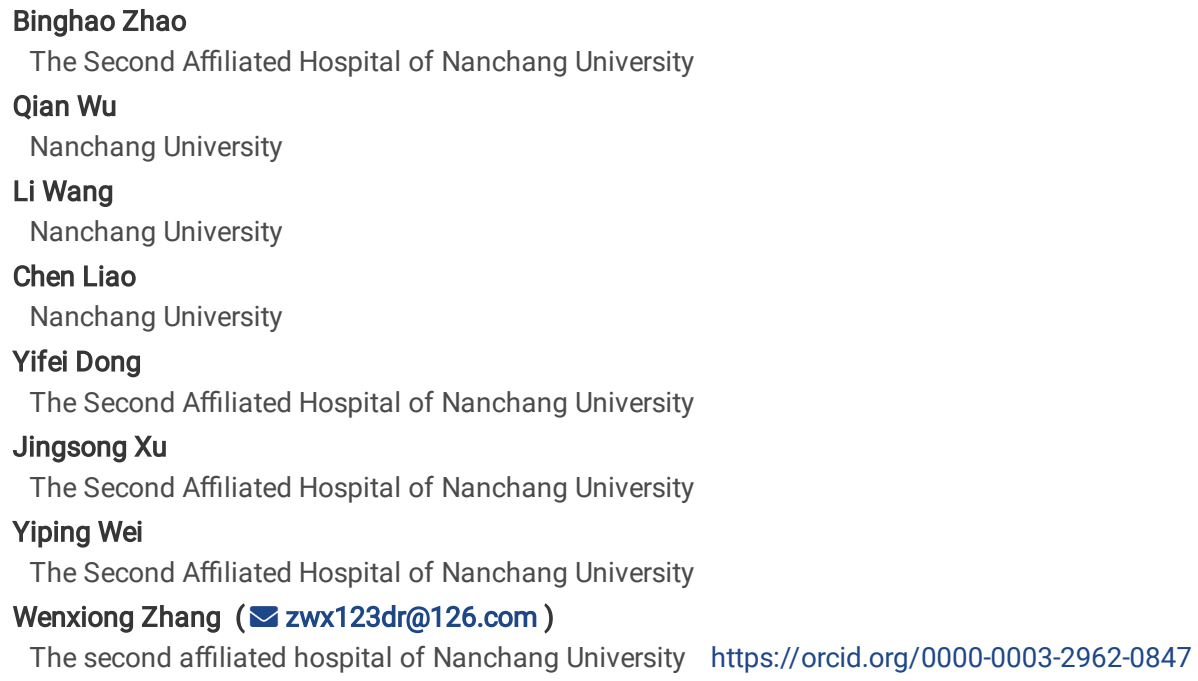




\section{Abstract \\ Background and aims}

Aspirin leads to substantial benefits for the secondary prevention of cardiovascular disease (CVD). We aimed to cast more light on aspirin's role for the primary prevention of CVD.

\section{Methods}

Databases were searched for clinical trials comparing aspirin vs. no aspirin use in this meta-analysis. Efficacy and safety profiles were rigorously investigated. Trial sequential analysis (TSA) was used to determine the robustness of the results.

\section{Results}

Fourteen studies with 163840 participants were eligible (mean follow-up 6.2 y). Aspirin intake was found to be associated with $9 \%, 13 \%$, and $12 \%$ reductions in the risk of cardiovascular events (CV events) (relative risk [RR]: 0.91, 95\% confidence intervals [Cl]: 0.87-0.96), myocardial infarction (RR: 0.87, 95\% Cl: $0.77-0.97$ ) and ischaemic stroke (RR: $0.88,95 \% \mathrm{Cl}: 0.80-0.96)$, respectively; aspirin intake was also associated with $40 \%, 30 \%$, and $57 \%$ increases in the risk of major bleeding (RR: 1.40, 95\% Cl: 1.29-1.53), intracranial bleeding (RR: 1.30, 95\% Cl: 1.11-1.52) and major gastrointestinal bleeding (RR: 1.57, 95\% Cl: $1.38-1.78$ ), respectively. Further, populations with low doses of aspirin intake ( $\leq 100 \mathrm{mg}$ ), populations $<65 \mathrm{y}$ old or populations with body mass index (BMI) $\geqq 25$ experienced more advantages; high-risk (10-y cardiovascular risk $\geqq 10 \%)$ and full diabetic individuals reported hardly clinical benefits.

\section{Conclusions}

Aspirin intake was associated with a reduced risk of CV events and an increased incidence of bleeding profiles in primary prevention. It is necessary to identify individual's CVD risk using clear examinations or assessments before aspirin intake, and truly realize individualized prescription.

\section{Introduction}

Currently, many patients are at high risk because their health is influenced by occlusive vascular disease; indeed, a long-term antiplatelet regimen (e.g., aspirin therapy) reduces the yearly risk of worse vascular events (such as nonfatal myocardial infarction, nonfatal stroke and vessel-related death) by almost onequarter ${ }^{1}$. Distinct benefits are observed with respect to the incidence of non-fatal cardiovascular events (CV events), with a small but definitive absolute risk reduction of approximately 10-20 CV events per 1000 per year. Despite the benefits of aspirin, the absolute risk of major gastrointestinal or other major extracranial bleeding is also increased by an order of magnitude, so in secondary prevention, the benefits exceed the risks ${ }^{2}$.

For primary prevention in patients without prior cardiovascular disease (CVD), both the risk without aspirin and absolute benefits of aspirin are smaller than those in secondary prevention. Although rates of death from coronary heart disease (CHD) and stroke in America have significantly decreased, CVD and cerebrovascular disease remain a large health and economic burden ${ }^{3}$. New guidelines suggest that regardless of bleeding risk, the wide use of aspirin is recommended for patients with a moderate risk of CHD, and a low dosage of aspirin ( $75-100 \mathrm{mg}$ daily) may be reasonably recommended to 40 - to 70 -year-old adults at high risk of CVD without increasing major bleeding (llb grade). New guidelines also recommended that age should be considered as a key determinant of the CVD risk, as a daily dose aspirin (alone or in combination with other drugs) has been recommended for all people above a specific age. Low doses of aspirin should not be recommended as primary prevention for 70-year-olds or for individuals with a high risk of bleeding ${ }^{3-11}$. However, a moderate risk of CVD is hard to define, and whether the high CVD risk populations as well as the diabetic populations can get real benefits from aspirin or not.

Deferring the start of long-term aspirin use for primary prevention is a noted alternative that has the main advantage of avoiding an increased risk of slight or major bleeding events but has the disadvantage that the initial manifestation may be a disabling or fatal event. In previous primary prevention trials ${ }^{12-25}$, control populations with non-fatal CVD (non-fatal CHD or non-fatal occlusive stroke) would probably be prescribed long-term aspirin use to avoid recurrence, hence helping to compare the efficacy of immediate versus deferred aspirin use.

A previous meta-analysis ${ }^{26}$ noted that aspirin reduced all-cause mortality, myocardial infarction (MI), and ischaemic stroke while increasing the risk of major bleeding; another pooled study ${ }^{27}$ showed that aspirin reduced nonfatal MI but did not significantly influence all-cause mortality. Above mentioned studies had heterogeneous results on all-cause mortality because they had involved different number of trials conducted in different time. Another key controversial point was on individuals' CVD risk classification that whether the higher risk individuals or the lower risk individuals could derive real prevention benefits from aspirin discussed by various guidelines or researchers. Actually, there are some meta-analysis discussing this topic emerging yearly, not so many addressed their "cost-effectiveness", which is to say if the conclusions are statistically sufficent and robust, no repetitive meta-analyses or further evidence are needed to some extent so that saving the cost on public health.

Given the large number of individuals affected by current studies and guidelines, and less helpful of the impact from no-innovative work on global health policy making, we conducted a comprehensive meta-analysis with the aim to resolve clinical controversial points under intention-to-treat principles and to evaluate the sufficiency of current synthesized evidence using trial sequential method. 


\section{Methods}

The current study was conducted in accordance with the Preferred Reporting Items for Systematic Reviews and Meta-Analysis (PRISMA) guidelines (Appendix Table S1). The protocol is available in PROSPERO (CRD42019127570).

\section{Data Source And Study Selection}

A rigorous search was performed in the PubMed, EMBASE, Cochrane Library, Web of Science and ClinicalTrials.gov databases from inception to February 1 , 2020, to retrieve randomized controlled trials (RCTs) relating to aspirin use in patients without prior CVD. The search had no language restrictions. The main key words used were "aspirin", "cardiovascular disease", "cardiovascular events", "coronary heart disease", and "randomized controlled trials". Reference lists of the eligible studies and identified meta-analyses were also reviewed (Appendix File S1).

The inclusion criteria were as follows: (1) enrolled adult participants ( $\geq 18 \mathrm{y}$ ) without preexisting CV events (CV events here include peripheral arterial disease, $\mathrm{CHD}$, prior myocardial infarction (MI), ischaemic stroke, prior percutaneous coronary intervention, prior coronary artery bypass grafting); (2) compared aspirin use to no aspirin use (placebo included); (3) had a follow-up no less than 1 year to confirm the high quality of primary studies; (4) provided reliable and available outcome data (at least one primary efficacy outcome of interest was reported); and (5) was an RCT.

Studies with the most comprehensive outcomes were included to avoid duplications; studies that assessed patients with diabetes but without atherosclerosis were also considered. JPAD ${ }^{19}$ and JPAD2 22 trials were both included for they had different characteristics and proportion of the incorporated individuals as well as the differed follow-up. We excluded pure basic studies, reviews, and animal experiments.

\section{Data Extraction And Outcome Definition}

Two authors (Binghao Zhao, Yiping Wei) independently performed the study screening and extracted the baseline characteristics of each eligible trial. The baseline characteristics included demographic characteristics of included populations, clinical information about the intervention/control arms, and essential outcome data as well as the study design. The adjusted hazard ratio (HR), odds ratio (OR) and relative risk (RR) of analysed outcomes were adjusted for fully adjusted models. If some studies used intention-to-treat principles, we extracted the intention-to-treat data. Any discrepancies between the reviewers were resolved by a third author. If there were any missing data, the original authors were contacted.

The primary efficacy outcomes were CV events, all-cause mortality and cardiovascular mortality due to their universal definitions and balance of efficacy and safety, which reduce heterogeneity among eligible studies. The secondary efficacy outcomes were all MI, total stroke, ischaemic stroke, cancer incidence and cancer mortality. The safety profile outcomes were major bleeding, intracranial bleeding and major gastrointestinal bleeding, as defined by each eligible trial. Intracranial bleeding was treated as a potential outcome of aspirin use in addition to CV events. All these definitions follow per included study's definition ${ }^{10}$.

Some studies even noted that aspirin increased the probability of cancer mortality, therefore, cancer outcomes were also appointed as exploratory outcome for robust evidence. The 10-y major adverse cardiovascular event rate (10-y MACE\%) was extracted and calculated by multiplying the annualized event rate for cardiovascular mortality, nonfatal MI, and nonfatal stroke. A 10-y MACE\% $\geq 10 \%$ was regarded as high risk; the others were regarded as low risk (Appendix File S1).

\section{Study Quality Assessment}

Methodological quality assessment was performed by three co-authors (Binghao Zhao, Li Wang, Wenxiong Zhang). We used the Cochrane Risk and Bias $\mathrm{Tool}^{28}$ recommended by the Cochrane handbook to evaluate the quality of each eligible study. There were several terms regarding the methodological quality of RCTs, and each study could be categorized as low, high or unclear quality; low-quality studies and those with unclear quality had a high risk of bias. Details are provided in the Appendix File S1.

\section{Statistical analysis}

For descriptive purposes and statistical convenience, weighted frequencies were calculated for categorical variables using the provided sample size of each trial. Multivariable RRs and $95 \%$ confidence intervals ( $95 \% \mathrm{Cls}$ ) as well as prediction intervals (represented by credible intervals, Crls) ${ }^{29}$ for primary/secondary efficacy outcomes of interest and primary safety outcomes were estimated using the DerSimonian-Laird (D-L) random effects model considering the existence of within- and between-study variability. For further statistical purposes, HRs and ORs were considered RRs in this study. Fully adjusted effect sizes (ESs) were logarithmically transformed to stabilize the variance; hence, the data distribution could be normalized.

Between-study heterogeneity and variability were quantified by Cochran's $Q$ test and $P^{2}$, whereby an $P>50 \%$ or a $P$-value for the $Q$ test $<0.10$ was considered to represent significant heterogeneity ${ }^{30}$. To provide more clinical implications, we conducted comprehensive subgroup analyses mainly focusing on several significant variables, including region, individuals' main age, mean body mass index (BMI), aspirin dose taken and 10-y MACE\%. For 10-y MACE\%, the computed value of 10-y MACE\% < $10 \%$ was defined as low risk, but the other populations were high risk. To provide more useful clinical data as well as to investigate the influence of individual studies on final results, we carried out sensitivity analyses by omitting one study each turn. 
Publication bias was assessed by funnel plots and Egger's test ${ }^{31}$, with $P<0.05$ indicating significant bias. All analyses were performed using $R$ project software (version 3.5.3, https://www.r-project.org/, USA) and other public packages (forest, ggplot2, survival, survminer etc.); a two-sided $P<0.05$ was considered statistically significant except where otherwise specified. More details are provided in the Appendix File S1.

\section{Trial Sequential Analysis}

Previous studies have confirmed that the risk of type 1 error from interim analyses can be reasonably reduced through monitoring boundaries and modifying the $P$-value. Similar in meta-analyses, random errors caused by sparse data and repetitive testing also enhance the risk of type 1 error. Such a method setting analogous trial sequential monitoring boundaries to meta-analyses is called trial sequential analysis (TSA), is used to determine whether evidence is reliable or conclusive 32,33 . Actually, random errors can be rectified and reduced using TSA software (version 0.9 beta (http://www.ctu.dk/tsa)) because it combines the estimation of the required information size (RIS) with an adjusted threshold for statistical significance. We assumed that if the Z-curve crossed the TSA boundary or entered the futility area, a sufficient effect was obtained, and further studies were not required; otherwise, the amount of evidence was considered insufficient. TSA was performed for a $10 \%$ relative risk reduction, conservatively, according to the TSA manual; there was also a $5 \%$ ( $a=0.05$; two-sided) risk of a type 1 error and $80 \%$ statistical power. Other parameters were set empirically following default settings.

\section{Results}

\section{Study selection and characteristics}

Among 1441 searched articles, we identified 26 studies for full-text review, of which 14 studies were eligible for qualitative and quantitative analyses (Appendix Figure S1). The 14 included studies ${ }^{12-25}$ encompassed a total of 163840 patients and used intention-to-treat principles. The detailed study characteristics are summarized in Table 1. 
Table 1

Characteristics of included studies and participants.

\begin{tabular}{|c|c|c|c|c|c|c|c|c|c|c|c|}
\hline Publication & $\begin{array}{l}\text { Study } \\
\text { population }\end{array}$ & $\begin{array}{l}\text { Number of } \\
\text { population }\end{array}$ & $\begin{array}{l}\text { Mean } \\
\text { age y/ } \\
\text { Male } \\
(\%)\end{array}$ & $\begin{array}{l}\text { Aspirin } \\
\text { use } \\
\text { (mg/day) }\end{array}$ & $\begin{array}{l}\text { Control } \\
\text { group }\end{array}$ & $\begin{array}{l}\text { Diabetes } \\
\text { No. (\%) }\end{array}$ & $\begin{array}{l}\text { Current } \\
\text { smokers } \\
\text { NO. (\%) }\end{array}$ & $\begin{array}{l}\text { Hypertension } \\
\text { NO. (\%) }\end{array}$ & $\begin{array}{l}\text { Mean } \\
\text { SBP } \\
\text { (mean } \\
\pm \text { SD) } \\
\text { mmHg }\end{array}$ & $\begin{array}{l}\text { Total } \\
\text { Cholesterol } \\
\text { (mean } \pm \\
\text { SD) } \\
\text { mmol/L }\end{array}$ & $B \wedge$ \\
\hline $\begin{array}{l}\text { Peto 1988; } \\
\text { UK, (BDS) }\end{array}$ & $\begin{array}{l}\text { Male } \\
\text { physicians }\end{array}$ & $\begin{array}{l}5139 \\
(3429 / 1710)\end{array}$ & $\begin{array}{l}61 / \\
5139 \\
(100)\end{array}$ & $\begin{array}{l}300 \text { or } \\
500\end{array}$ & $\begin{array}{l}\text { No } \\
\text { aspirin }\end{array}$ & $101(2)$ & $661(13)$ & $508(10)$ & $\begin{array}{l}136 \pm \\
17\end{array}$ & NA & $\begin{array}{l}24 \\
\pm 2\end{array}$ \\
\hline $\begin{array}{l}\text { Steering } \\
\text { 1989; } \\
\text { America, } \\
\text { (PHS) }^{13}\end{array}$ & $\begin{array}{l}\text { Male } \\
\text { physicians }\end{array}$ & $\begin{array}{l}22071 \\
(11037 / 11034)\end{array}$ & $\begin{array}{l}53 / \\
22071 \\
(100)\end{array}$ & 325 & Placebo & $533(2)$ & $\begin{array}{l}2438 \\
(11)\end{array}$ & 5297 (24) & $\begin{array}{l}126 \pm \\
12\end{array}$ & $5.5 \pm 1.2$ & $\begin{array}{l}24 \\
\pm \Xi\end{array}$ \\
\hline $\begin{array}{l}\text { Meade 1998; } \\
\text { UK, (TPT) }{ }^{14}\end{array}$ & $\begin{array}{l}\text { Males in the } \\
\text { top } 20-25 \% \\
\text { risk of CV } \\
\text { events }\end{array}$ & $\begin{array}{l}2540 \\
(1268 / 1272)^{c}\end{array}$ & $\begin{array}{l}57 / \\
2540 \\
(100)\end{array}$ & 75 & Placebo & $51(2)$ & $83(3)$ & 278 (11) & $\begin{array}{l}139 \pm \\
18\end{array}$ & $6.4 \pm 1.0$ & $\begin{array}{l}27 \\
\pm \Xi\end{array}$ \\
\hline $\begin{array}{l}\text { Hansson } \\
\text { 1998; multi- } \\
\text { nations, } \\
\left(\text { HOT) }{ }^{15}\right.\end{array}$ & $\begin{array}{l}\text { Hypertensive } \\
\text { populations }\end{array}$ & $\begin{array}{l}18790 \\
(9399 / 9391)\end{array}$ & $\begin{array}{l}61 / \\
9959 \\
(53)\end{array}$ & 75 & Placebo & 1503 (8) & $\begin{array}{l}2988 \\
(16)\end{array}$ & $18790(100)$ & $\begin{array}{l}170 \pm \\
14\end{array}$ & $6.0 \pm 1.1$ & $\begin{array}{l}28 \\
\pm<\end{array}$ \\
\hline $\begin{array}{l}\text { De Gaetano } \\
\text { 2001; Italy, } \\
(P P P)^{16}\end{array}$ & $\begin{array}{l}\text { Populations } \\
\text { with } \geq 1 \mathrm{CV} \\
\text { risk factor }\end{array}$ & $\begin{array}{l}4495 \\
(2226 / 2269)\end{array}$ & $\begin{array}{l}64 / \\
1912 \\
(42)\end{array}$ & 100 & $\begin{array}{l}\text { No } \\
\text { aspirin }\end{array}$ & 742 (17) & $667(15)$ & 3065 (68) & $\begin{array}{l}145 \pm \\
16\end{array}$ & $6.1 \pm 1.2$ & $\begin{array}{l}27 \\
\pm<\end{array}$ \\
\hline $\begin{array}{l}\text { Ridker 2005; } \\
\text { America, } \\
(\mathrm{WHS})^{17}\end{array}$ & $\begin{array}{l}\text { Healthy } \\
\text { females }\end{array}$ & $\begin{array}{l}39876 \\
(19934 / 19942)\end{array}$ & $\begin{array}{l}54 / 0 \\
(0)\end{array}$ & 100 & Placebo & 1037 (3) & $\begin{array}{l}5224 \\
(13)\end{array}$ & $10328(26)$ & NA & $5.2 \pm 1.0$ & $\begin{array}{l}26 \\
\pm 5\end{array}$ \\
\hline $\begin{array}{l}\text { Belch 2008; } \\
\text { UK, } \\
\text { (POPADAD) }^{18}\end{array}$ & $\begin{array}{l}\text { Diabetic } \\
\text { populations } \\
(\mathrm{ABPI} \leq 0.99)\end{array}$ & $\begin{array}{l}1276 \\
(638 / 638)\end{array}$ & $\begin{array}{l}60 / \\
563 \\
(44)\end{array}$ & 100 & Placebo & $\begin{array}{l}1276 \\
(100)\end{array}$ & NA & NA & $\begin{array}{l}145 \pm \\
21\end{array}$ & 5.5 & 29 \\
\hline $\begin{array}{l}\text { Ogawa et al, } \\
\text { 2008; Japan, } \\
(\text { JPAD) }\end{array}$ & $\begin{array}{l}\text { Diabetic } \\
\text { populations }\end{array}$ & $\begin{array}{l}2539 \\
(1262 / 1277)\end{array}$ & $\begin{array}{l}65 / \\
1387 \\
(55)\end{array}$ & 81 or 100 & $\begin{array}{l}\text { No } \\
\text { aspirin }\end{array}$ & $\begin{array}{l}2539 \\
(100)\end{array}$ & $537(21)$ & $1473(58)$ & $\begin{array}{l}135 \pm \\
15\end{array}$ & $5.2 \pm 0.9$ & $\begin{array}{l}24 \\
\pm<\end{array}$ \\
\hline $\begin{array}{l}\text { Fowkes 2010; } \\
\text { UK, (AAA) }\end{array}$ & $\begin{array}{l}\text { Populations } \\
\text { with } \leq 0.95 \\
\text { ABPI }\end{array}$ & $\begin{array}{l}3350 \\
(1675 / 1675)\end{array}$ & $\begin{array}{l}62 / \\
954 \\
(28)\end{array}$ & 100 & Placebo & $88(3)$ & $\begin{array}{l}1085 \\
(32)\end{array}$ & NA & $\begin{array}{l}148 \pm \\
22\end{array}$ & $6.2 \pm 1.1$ & $\mathrm{~N} /$ \\
\hline $\begin{array}{l}\text { Ikeda 2014; } \\
\text { Japan. } \\
\text { (JPPP) }^{21}\end{array}$ & $\begin{array}{l}\text { Hypertensive, } \\
\text { hyperlipidemic } \\
\text { or diabetic } \\
\text { populations }\end{array}$ & $\begin{array}{l}14464 \\
(7220 / 7244)\end{array}$ & $\begin{array}{l}71 / \\
6123 \\
(42)\end{array}$ & 100 & $\begin{array}{l}\text { No } \\
\text { aspirin }\end{array}$ & $\begin{array}{l}4903 \\
(34)\end{array}$ & $\begin{array}{l}1893 \\
(13)\end{array}$ & $12278(85)$ & $\begin{array}{l}137 \pm \\
16\end{array}$ & $5.3 \pm 0.8$ & $\begin{array}{l}24 \\
\pm \Xi\end{array}$ \\
\hline $\begin{array}{l}\text { Saito et al, } \\
\text { 2017; Japan, } \\
\left(\text { JPAD2) }{ }^{22}\right.\end{array}$ & $\begin{array}{l}\text { Diabetic } \\
\text { populations }\end{array}$ & $\begin{array}{l}2160 \\
(992 / 1168)\end{array}$ & $\begin{array}{l}65 / \\
1195 \\
(55)\end{array}$ & 81 or 100 & $\begin{array}{l}\text { No } \\
\text { aspirin }\end{array}$ & $\begin{array}{l}2160 \\
(100)\end{array}$ & 459 (21) & $2142(58)$ & $\begin{array}{l}135 \pm \\
15\end{array}$ & $5.2 \pm 0.9$ & $\begin{array}{l}24 \\
\pm<\end{array}$ \\
\hline $\begin{array}{l}\text { Bowman } \\
\text { 2018; UK, } \\
(\text { ASCEND) }\end{array}$ & $\begin{array}{l}\text { Diabetic } \\
\text { populations }\end{array}$ & $\begin{array}{l}15480 \\
(7740 / 7740)\end{array}$ & $\begin{array}{l}63 / \\
9684 \\
(63)\end{array}$ & 100 & Placebo & $\begin{array}{l}15480 \\
(100)\end{array}$ & 1279 (8) & $9533(62)$ & $\begin{array}{l}136 \pm \\
15\end{array}$ & $4.2 \pm 0.9$ & $\begin{array}{l}30 \\
\pm \epsilon\end{array}$ \\
\hline $\begin{array}{l}\text { Gaziano } \\
\text { 2018; multi- } \\
\text { nations, } \\
\text { (ARRIVE) }\end{array}$ & $\begin{array}{l}\text { Males with } \geq \\
2 \text { and females } \\
\text { with } \geq 3 \mathrm{CV} \\
\text { risk factors, } \\
\text { with } 10-20 \% \\
10-y \text { MACE } \\
\text { risk }\end{array}$ & $\begin{array}{l}12546 \\
(6270 / 6276)\end{array}$ & $\begin{array}{l}64 / \\
8838 \\
(70)\end{array}$ & 100 & Placebo & $0(0)$ & $\begin{array}{l}3594 \\
(29)\end{array}$ & $7866(63)$ & $\begin{array}{l}144 \\
(90- \\
199)^{\mathrm{e}}\end{array}$ & NA & $\begin{array}{l}28 \\
\pm<\end{array}$ \\
\hline $\begin{array}{l}\text { McNeil 2018; } \\
\text { multi-nations, } \\
(\text { ASPREE) }\end{array}$ & $\begin{array}{l}\geq 65 y \\
\text { populations }\end{array}$ & $\begin{array}{l}19114 \\
(9525 / 9589)\end{array}$ & $\begin{array}{l}74 / \\
8331 \\
(44)\end{array}$ & 100 & Placebo & $\begin{array}{l}2057 \\
(11)\end{array}$ & $735(4)$ & $14283(74)$ & $\begin{array}{l}140 \pm \\
17\end{array}$ & $5.3 \pm 1.0$ & $\begin{array}{l}28 \\
\pm 4\end{array}$ \\
\hline
\end{tabular}

Abbreviations: SBP: systolic blood pressure; BMI: body mass index; MACE: major adverse cardiovascular events; CV risk: cardiovascular risk; ABPI: ankle-brac myocardial infraction; NA: not available.

Two studies ${ }^{13,17}$ were conducted in America, 6 studies were conducted in Europe $\left(5^{12,14,18,20,23}\right.$ in the UK and $1^{16}$ in Italy), 3 studies ${ }^{19,21,22}$ were performed in Japan, and 3 studies ${ }^{15,24,25}$ were performed in multiple nations. The comparator treatment was a placebo group in 9 studies ${ }^{13-15,17,18,20,23-25}$ and was a no aspirin group in 5 studies. Of note, in addition to aspirin and placebo, 6 studies used a factorial design, in which $1^{14}$ study used warfarin, $2^{16,17}$ used vitamin $\mathrm{E}, 1^{23}$ prescribed $\mathrm{n}-3$ fatty acid, $1^{18}$ used antioxidants, and $1^{12}$ supplied anti-hypertension drugs. Three studies ${ }^{12-14}$ exclusively enrolled male individuals ( 29 750 males), and 1 study ${ }^{17}$ specially enrolled female individuals (39 876 females). Across the included studies, 78696 (48\%) patients were males. Four studies $^{18,19,22,23}$ exclusively enrolled diabetic patients (including type I and type II diabetes). The mean BMI of eligible participants was 28.5 , and the mean 
10-y MACE\% was 7.24. The median duration was 8.1 y $\left(4^{16}\right.$ to $\left.13^{14,22}\right)$, and the mean follow-up was 6.2 y. The studies were published between $1988^{12}$ and $2018^{23-25}$. All studies were written in English, and there was no attempt to ask the primary authors for raw data.

\section{Methodological quality assessment}

Of the 14 included studies, 9 studies used double-blind methods and 5 studies ${ }^{12,16,19,21,22}$ used open-label settings. Three studies ${ }^{13,14,16}$ had selective reporting or other bias. Of the included studies, $7^{15,17,18,20,23-25}$ were of low risk and 712-14, 16, 19, 21,22 were of high risk (Appendix Figure S2 and Appendix Table S2).

\section{The Primary Efficacy Outcomes}

For the primary efficacy outcomes, twelve studies ${ }^{12,13,15-17,19-25}$ involving 160024 individuals reported CV event outcomes, and we found that the use of aspirin was associated with a 9\% reduction in CV events (RR: 0.91, 95\% Cl: 0.87-0.96; P < 0.001; prediction interval: 0.91, 95\% Crl: 0.86-0.96) compared to no aspirin use, and there was no significant heterogeneity $\left(I^{2}=0 ; P=0.64\right)$. Thirteen studies ${ }^{12-21,23-25}$ including 161680 individuals examined all-cause mortality outcomes; aspirin use did not lead to a significant reduction in all-cause mortality (RR: 0.97, 95\% Cl: 0.93-1.02; P=0.22; prediction interval: 0.97, 95\% Crl: $0.92-1.02)$, and there was no heterogeneity $\left(I^{2}=0 ; P=0.60\right)$. Fourteen studies ${ }^{12-25}$ (163 840 participants) examined cardiovascular mortality; aspirin use was not significantly associated with cardiovascular mortality reduction (RR: $0.95,95 \% \mathrm{Cl}: 0.87-1.03 ; \mathrm{P}=0.23$; Prediction interval: $0.95,95 \%$ Crl: $0.86-1.04$ ), and there was no significant heterogeneity $\left(I^{2}=0 ; P=0.57\right)$ (Fig. 1).

\section{The Secondary Efficacy Outcomes}

Regarding the secondary efficacy outcomes, fourteen studies ${ }^{12-25}$ with 163840 individuals revealed that aspirin intake was associated with a $13 \%$ reduction in all Mls (RR: $0.87,95 \% \mathrm{Cl}: 0.77-0.97 ; \mathrm{P}=0.02$; prediction interval: $0.87,95 \% \mathrm{Crl}: 0.60-1.26)$, and there was significant heterogeneity $\left(\mathrm{I}^{2}=58 \%\right.$; $\mathrm{P}<0.01$ ). Eleven studies ${ }^{12-14,16,17,19-23,25}$ (131 228 individuals) revealed that aspirin intake was associated with a $12 \%$ risk reduction in ischaemic stroke (RR: 0.88 , 95\% Cl: 0.80-0.96; $\mathrm{P}<0.01$; prediction interval: $0.88,95 \% \mathrm{Crl}: 0.79-0.98)$, and there was no significant heterogeneity $\left(\mathrm{I}^{2}=0 ; \mathrm{P}=0.62\right)$. Fourteen studies ${ }^{12-25}$ (163 840 individuals) revealed that aspirin use was not significantly associated with total stroke (RR: $0.94,95 \% \mathrm{Cl}$ : $0.88-1.02 ; \mathrm{P}=0.13 ; \mathrm{Prediction}$ interval: $0.94,95 \%$ Crl: $0.87-1.02)$, and there was no significant heterogeneity $\left(I^{2}=0 ; P=0.59\right)$.

Furthermore, we explored the cancer outcomes. Ten studies ${ }^{12,15-21,23,25}$ including 124523 participants and 12 studies ${ }^{12-21,23,25}$ including 149134 participants reported cancer incidence and cancer mortality, respectively. There was no significant difference in cancer incidence (RR: 1.00, 95\% Cl: 0.95-1.06; $\mathrm{P}=0.87$; prediction interval: $1.00,95 \% \mathrm{Crl}: 0.88-1.15)$ or cancer mortality (RR: $1.03,95 \% \mathrm{Cl}: 0.94-1.12 ; \mathrm{P}=0.87$; prediction interval: $1.03,95 \%$ Crl: $0.86-1.22)$ between the aspirin use and no aspirin use groups, and there was no significant heterogeneity $\left(I^{2}=36 \%, P=0.12 ; I^{2}=21 \%, P=0.24\right.$, respectively). Aspirin showed the potential to increase the risk of cancer mortality (Appendix Figure S3).

\section{The safety profile outcomes}

Safety profiles outcomes included major bleeding, intracranial bleeding and major gastrointestinal bleeding. Twelve studies ${ }^{12-17,19-23,25}$ including 150397 patients examined major bleeding events; aspirin use was found to significantly increase the risk of major bleeding by $40 \%$ (RR: $1.40,95 \% \mathrm{Cl}$ : $1.29-1.53$; $\mathrm{P}<$ 0.01; prediction interval: $1.40,95 \% \mathrm{Crl}: 1.27-1.54)$, and there was no significant heterogeneity $\left(\mathrm{I}^{2}=0 \% ; \mathrm{P}=0.54\right)$. Thirteen studies ${ }^{12-17,19-25}(162934$ participants) examined intracranial bleeding; aspirin use was associated with a 30\% increase in intracranial bleeding (RR: 1.30, 95\% Cl: 1.11-1.52; P < 0.01; prediction interval: $1.30,95 \% \mathrm{Crl}: 1.09-1.55)$, and there was no heterogeneity $\left(\mathrm{I}^{2}=0 \% ; \mathrm{P}=0.84\right)$. Eleven trials ${ }^{13-17,19,20,22-25}(143340$ participants) examined major gastrointestinal bleeding; aspirin intake was associated with a 57\% increase in major gastrointestinal bleeding (RR: 1.57, 95\% Cl: 1.38-1.78; P< 0.01; prediction interval: $1.57,95 \% \mathrm{Crl}: 1.36-1.82)$, and there was no heterogeneity $\left(\mathrm{I}^{2}=0 \% ; \mathrm{P}=0.57\right)$. The finding that aspirin use significantly increased the risk of bleeding events led us to identify the proper indicators for balancing the benefits and harm of clinical routines (Figure 2).

\section{Subgroup analysis for further clinical implications}

Subgroups involving region, mean age, mean BMI, aspirin dosage in the intervention arm and 10-y MACE\% were constructed, and subgroup analyses were performed (Table 2). We observed that populations with a dosage of $\leq 100 \mathrm{mg} / \mathrm{d}$ experienced more benefits with respect to CV events, Ml, total stroke and ischaemic stroke than those with a dosage $>100 \mathrm{mg} / \mathrm{d}$. Individuals with a BMI $\geqq 25$ seemed experience more aspirin-induced benefits with respect to cardiovascular and cerebrovascular outcomes (CV events, RR: 0.91, 95\% Cl: 0.86-0.98; total stroke, RR: 0.90, 95\% Cl: 0.82-0.99; ischaemic stroke, RR: 0.85, 95\% $\mathrm{Cl}: 0.76-0.95)$ than individuals with a $\mathrm{BMI}<25$ with similar bleeding events. Aspirin-induced cardiovascular benefits were consistently found in participants with a mean age $<65 \mathrm{y}$; however, they were not as robust in the patients with a mean age $\geq 65 \mathrm{y}$, with only one statistically significant outcome for CV events (RR: $0.90,95 \% \mathrm{Cl}: 0.81-1.00$ ). Participants with a low 10-y MACE\% risk had the potential to obtain more cardiovascular advantages from aspirin use than those with a high 10-y MACE\% risk. There was no significant difference in cardiovascular outcomes and bleeding events between patients from different regions. Across the subgroup analyses, aspirin still had no statistically significant effects on cancer incidence or mortality. All of the above results are presented in Table 2. 
Table 2

Summarized results of total and subgroup analyses.

\begin{tabular}{|c|c|c|c|c|c|c|c|c|c|c|c|c|}
\hline \multirow[t]{2}{*}{ Items/Outcomest } & \multirow[t]{2}{*}{ Total } & \multicolumn{4}{|l|}{ By region } & \multicolumn{2}{|c|}{$\begin{array}{l}\text { By mean age } \\
\text { (V) }\end{array}$} & \multicolumn{2}{|c|}{ By mean BMI } & \multicolumn{2}{|c|}{$\begin{array}{l}\text { By aspirin } \\
\text { dose (mg) }\end{array}$} & \multirow{2}{*}{$\begin{array}{l}\text { By } 10 \\
\text { MACE } \\
\begin{array}{l}\text { Low } \\
\text { risk }\end{array}\end{array}$} \\
\hline & & $\begin{array}{l}\text { North } \\
\text { America }\end{array}$ & Europe & Asia & $\begin{array}{l}\text { Multiple } \\
\text { nations }\end{array}$ & $<65$ & $\geqq 65$ & $<25$ & $\geqq 25$ & $\leq$ & $>100$ & \\
\hline CV events & $\begin{array}{l}0.91 \\
(0.87- \\
0.96)\end{array}$ & $\begin{array}{l}0.88 \\
(0.80- \\
0.97)\end{array}$ & $\begin{array}{l}0.94 \\
(0.86- \\
1.03)\end{array}$ & $\begin{array}{l}0.97 \\
(0.85- \\
1.10)\end{array}$ & $\begin{array}{l}0.90 \\
(0.82- \\
0.98)\end{array}$ & $\begin{array}{l}0.92 \\
(0.87- \\
0.97)\end{array}$ & $\begin{array}{l}0.90 \\
(0.81- \\
1.00)\end{array}$ & $\begin{array}{l}0.91 \\
(0.84- \\
0.99)\end{array}$ & $\begin{array}{l}0.91 \\
(0.86- \\
0.98)\end{array}$ & $\begin{array}{l}0.92 \\
(0.87- \\
0.97)\end{array}$ & $\begin{array}{l}0.91 \\
(0.75- \\
1.10)\end{array}$ & $\begin{array}{l}0.89 \\
(0.84- \\
0.96)\end{array}$ \\
\hline $\begin{array}{l}\text { All-cause } \\
\text { mortality }\end{array}$ & $\begin{array}{l}0.97 \\
(0.93- \\
1.02)\end{array}$ & $\begin{array}{l}0.95 \\
(0.87- \\
1.05\end{array}$ & $\begin{array}{l}0.94 \\
(0.88- \\
1.01\end{array}$ & $\begin{array}{l}0.98 \\
(0.84- \\
1.13)\end{array}$ & $\begin{array}{l}1.03 \\
(0.91- \\
1.17)\end{array}$ & $\begin{array}{l}0.95 \\
(0.90- \\
1.00)\end{array}$ & $\begin{array}{l}1.06 \\
(0.95- \\
1.18)\end{array}$ & $\begin{array}{l}0.94 \\
(0.87- \\
1.03\end{array}$ & $\begin{array}{l}0.99 \\
(0.92- \\
1.06)\end{array}$ & $\begin{array}{l}0.98 \\
(0.93- \\
1.03\end{array}$ & $\begin{array}{l}0.93 \\
(0.81- \\
1.06)\end{array}$ & $\begin{array}{l}1.00 \\
(0.92- \\
1.08)\end{array}$ \\
\hline $\begin{array}{l}\text { Cardiovascular } \\
\text { mortality }\end{array}$ & $\begin{array}{l}0.95 \\
(0.87- \\
1.03)\end{array}$ & $\begin{array}{l}0.96 \\
(0.79- \\
1.17)\end{array}$ & $\begin{array}{l}0.97 \\
(0.85- \\
1.11)\end{array}$ & $\begin{array}{l}0.76 \\
(0.31- \\
1.90)\end{array}$ & $\begin{array}{l}0.90 \\
(0.77- \\
1.07)\end{array}$ & $\begin{array}{l}0.96 \\
(0.88- \\
1.06)\end{array}$ & $\begin{array}{l}0.82 \\
(0.53- \\
1.29)\end{array}$ & $\begin{array}{l}0.97 \\
(0.84- \\
1.12\end{array}$ & $\begin{array}{l}0.92 \\
(0.83- \\
1.03\end{array}$ & $\begin{array}{l}0.94 \\
(0.85- \\
1.03)\end{array}$ & $\begin{array}{l}0.99 \\
(0.80- \\
1.23\end{array}$ & $\begin{array}{l}0.91 \\
(0.79- \\
1.04)\end{array}$ \\
\hline All MI & $\begin{array}{l}0.87 \\
(0.77- \\
0.97)\end{array}$ & $\begin{array}{l}0.78 \\
(0.45- \\
1.34\end{array}$ & $\begin{array}{l}0.95 \\
(0.86- \\
1.05)\end{array}$ & $\begin{array}{l}0.89 \\
(0.69- \\
1.16)\end{array}$ & $\begin{array}{l}0.81 \\
(0.66- \\
1.01\end{array}$ & $\begin{array}{l}0.87 \\
(0.76- \\
1.00)\end{array}$ & $\begin{array}{l}0.90 \\
(0.75- \\
1.08)\end{array}$ & $\begin{array}{l}0.78 \\
(0.61- \\
0.99)\end{array}$ & $\begin{array}{l}0.93 \\
(0.86- \\
1.02)\end{array}$ & $\begin{array}{l}0.91 \\
(0.83- \\
0.99)\end{array}$ & $\begin{array}{l}0.78 \\
(0.44- \\
1.38)\end{array}$ & $\begin{array}{l}0.81 \\
(0.66- \\
1.00)\end{array}$ \\
\hline Total stroke & $\begin{array}{l}0.94 \\
(0.88- \\
1.02)\end{array}$ & $\begin{array}{l}0.99 \\
(0.69- \\
1.43)\end{array}$ & $\begin{array}{l}0.89 \\
(0.78- \\
1.01\end{array}$ & $\begin{array}{l}0.99 \\
(0.82- \\
1.18)\end{array}$ & $\begin{array}{l}1.00 \\
(0.87- \\
1.14)\end{array}$ & $\begin{array}{l}0.94 \\
(0.86- \\
1.02)\end{array}$ & $\begin{array}{l}0.97 \\
(0.84- \\
1.13)\end{array}$ & $\begin{array}{l}1.04 \\
(0.92- \\
1.17)\end{array}$ & $\begin{array}{l}0.90 \\
(0.82- \\
0.99)\end{array}$ & $\begin{array}{l}0.92 \\
(0.85- \\
1.00)\end{array}$ & $\begin{array}{l}1.16 \\
(0.94- \\
1.44\end{array}$ & $\begin{array}{l}0.97 \\
(0.86- \\
1.11)\end{array}$ \\
\hline Ischaemic stroke & $\begin{array}{l}0.88 \\
(0.80- \\
0.96)\end{array}$ & $\begin{array}{l}0.91 \\
(0.64- \\
1.29)\end{array}$ & $\begin{array}{l}0.89 \\
(0.76- \\
1.03)\end{array}$ & $\begin{array}{l}0.88 \\
(0.71- \\
1.10)\end{array}$ & $\begin{array}{l}0.89 \\
(0.72- \\
1.11)\end{array}$ & $\begin{array}{l}0.88 \\
(0.78- \\
1.00)\end{array}$ & $\begin{array}{l}0.88 \\
(0.74- \\
1.04)\end{array}$ & $\begin{array}{l}0.98 \\
(0.82- \\
1.16)\end{array}$ & $\begin{array}{l}0.85 \\
(0.76- \\
0.95)\end{array}$ & $\begin{array}{l}0.85 \\
(0.78- \\
0.94)\end{array}$ & $\begin{array}{l}1.14 \\
(0.86- \\
1.52)\end{array}$ & $\begin{array}{l}0.87 \\
(0.76- \\
0.98)\end{array}$ \\
\hline Cancer incidence & $\begin{array}{l}1.00 \\
(0.95- \\
1.06)\end{array}$ & $\begin{array}{l}1.01 \\
(0.94- \\
1.08)\end{array}$ & $\begin{array}{l}0.98 \\
(0.91- \\
1.06)\end{array}$ & $\begin{array}{l}1.06 \\
(0.79- \\
1.42)\end{array}$ & $\begin{array}{l}1.01 \\
(0.94- \\
1.09)\end{array}$ & $\begin{array}{l}0.99 \\
(0.94- \\
1.04)\end{array}$ & $\begin{array}{l}1.05 \\
(0.92- \\
1.21)\end{array}$ & $\begin{array}{l}1.02 \\
(0.88- \\
1.19)\end{array}$ & $\begin{array}{l}1.01 \\
(0.97- \\
1.06)\end{array}$ & $\begin{array}{l}1.02 \\
(0.96- \\
1.07)\end{array}$ & $\begin{array}{l}0.91 \\
(0.77- \\
1.08)\end{array}$ & $\begin{array}{l}1.05 \\
(0.98- \\
1.13)\end{array}$ \\
\hline Cancer mortality & $\begin{array}{l}1.03 \\
(0.94- \\
1.12)\end{array}$ & $\begin{array}{l}1.00 \\
(0.84- \\
1.18)\end{array}$ & $\begin{array}{l}0.94 \\
(0.84- \\
1.05)\end{array}$ & $\begin{array}{l}1.07 \\
(0.88- \\
1.30)\end{array}$ & $\begin{array}{l}1.18 \\
(0.94- \\
1.48)\end{array}$ & $\begin{array}{l}0.97 \\
(0.89- \\
1.05)\end{array}$ & $\begin{array}{l}1.19 \\
(1.04- \\
1.36)\end{array}$ & $\begin{array}{l}1.03 \\
(0.90- \\
1.18)\end{array}$ & $\begin{array}{l}1.04 \\
(0.91- \\
1.19)\end{array}$ & $\begin{array}{l}1.03 \\
(0.95- \\
1.12)\end{array}$ & $\begin{array}{l}0.97 \\
(0.68- \\
1.40)\end{array}$ & $\begin{array}{l}1.11 \\
(0.96- \\
1.27)\end{array}$ \\
\hline Major bleeding & $\begin{array}{l}1.40 \\
(1.29- \\
1.53)\end{array}$ & $\begin{array}{l}1.44 \\
(1.15- \\
1.82)\end{array}$ & $\begin{array}{l}1.46 \\
(1.10- \\
1.95\end{array}$ & $\begin{array}{l}1.35 \\
(1.10- \\
1.67)\end{array}$ & $\begin{array}{l}1.49 \\
(1.18- \\
1.88)\end{array}$ & $\begin{array}{l}1.39 \\
(1.21- \\
1.59)\end{array}$ & $\begin{array}{l}1.42 \\
(1.25- \\
1.62)\end{array}$ & $\begin{array}{l}1.47 \\
(1.26- \\
1.71)\end{array}$ & $\begin{array}{l}1.36 \\
(1.21- \\
1.53)\end{array}$ & $\begin{array}{l}1.39 \\
(1.28- \\
1.52)\end{array}$ & $\begin{array}{l}1.40 \\
(0.92- \\
2.12)\end{array}$ & $\begin{array}{l}1.42 \\
(1.27- \\
1.60)\end{array}$ \\
\hline $\begin{array}{l}\text { Intracranial } \\
\text { bleeding }\end{array}$ & $\begin{array}{l}1.30 \\
(1.11- \\
1.52)\end{array}$ & $\begin{array}{l}1.40 \\
(0.96- \\
2.05)\end{array}$ & $\begin{array}{l}1.26 \\
(0.91- \\
1.74)\end{array}$ & $\begin{array}{l}1.21 \\
(0.82- \\
1.77)\end{array}$ & $\begin{array}{l}1.18 \\
(0.77- \\
1.80)\end{array}$ & $\begin{array}{l}1.18 \\
(0.96- \\
1.47)\end{array}$ & $\begin{array}{l}1.46 \\
(1.15- \\
1.84)\end{array}$ & $\begin{array}{l}1.25 \\
(0.95- \\
1.65)\end{array}$ & $\begin{array}{l}1.31 \\
(1.08- \\
1.60)\end{array}$ & $\begin{array}{l}1.28 \\
(1.08- \\
1.51)\end{array}$ & $\begin{array}{l}1.57 \\
(0.89- \\
2.77\end{array}$ & $\begin{array}{l}1.40 \\
(1.15- \\
1.70)\end{array}$ \\
\hline $\begin{array}{l}\text { Major } \\
\text { gastrointestinal } \\
\text { bleeding }\end{array}$ & $\begin{array}{l}1.57 \\
(1.38- \\
1.78)\end{array}$ & $\begin{array}{l}1.47 \\
(1.17- \\
1.86)\end{array}$ & $\begin{array}{l}1.61 \\
(1.02- \\
2.54)\end{array}$ & $\begin{array}{l}1.87 \\
(1.02- \\
3.44)\end{array}$ & $\begin{array}{l}1.72 \\
(1.40- \\
2.11)\end{array}$ & $\begin{array}{l}1.58 \\
(1.35- \\
1.85)\end{array}$ & $\begin{array}{l}1.58 \\
(1.24- \\
2.01)\end{array}$ & $\begin{array}{l}1.92 \\
(1.47- \\
2.51)\end{array}$ & $\begin{array}{l}1.49 \\
(1.28- \\
1.72)\end{array}$ & $\begin{array}{l}1.55 \\
(1.36- \\
1.77)\end{array}$ & $\begin{array}{l}1.75 \\
(1.10- \\
2.78)\end{array}$ & $\begin{array}{l}1.57 \\
(1.33- \\
1.85)\end{array}$ \\
\hline
\end{tabular}

Abbreviations: BMI: body mass index; MACE: major adverse cardiovascular event rate; CV event: cardiovascular event; MI: myocardial infraction.

${ }^{*} \mathrm{~A} 10-\mathrm{y}$ MACE$\%$ of at least $10 \%$ was regarded as high CV risk and less than $10 \%$ was low; ${ }^{\dagger}$ All the outcomes were shown in RR and $95 \% \mathrm{Cl}$ form.

\section{Sensitivity analysis}

In sensitivity analyses, many variables were classified into different subgroups. To better eliminate bias and heterogeneous interactions (TPT ${ }^{14}$ trial was excluded for warfarin use), we used the inverse variance (IV) statistical method. Most of the results were consistent with the primary results and remained robust through sensitivity analyses. Interestingly, we observed increased aspirin-induced benefits for cardiac outcomes (CV events, RR: 0.90, 95\% Cl: 0.85-0.95; all MI, RR: $0.83,95 \% \mathrm{Cl}: 0.72-0.96$; ischaemic stroke, RR: $0.86,95 \% \mathrm{Cl}: 0.76-0.97$ ) among trials with diabetic and nondiabetic patients compared to the trials involving only diabetic patients. We also observed aspirin-induced benefits when excluding patients with asymptomatic peripheral artery disease (PAD). Furthermore, after excluding trials published before 2000, the cardiovascular benefits were still obvious. No effects on cancer were found across sensitivity analyses (Table 3). The omission process as well as the results of the heterogeneity analyses can be found in Table $\mathbf{3}$ and Appendix File S2-S12.

These findings implied that aspirin use among diabetic individuals may not lead to the primary prevention of CVD because diabetes, which is known as a risk factor for CVD, might indirectly enhance the CV risk estimated by the MACE; similarly, the efficacy of aspirin use in studies including both diabetic and nondiabetic patients was excellent. Second, diagnosis technology is developing over time, which means that more patients with potential or asymptomatic CVD could be properly diagnosed and excluded before entering clinical trials or taking aspirin for "primary prevention". Therefore, the preferable role of aspirin in the primary prevention of CVD would be highlighted, especially in recently published studies (after 2000). Finally, early screening for PAD was equally important to help identify individuals who may not benefit from aspirin. 
Table 3

Summarized results of the sensitivity analysis.

\begin{tabular}{|c|c|c|c|c|c|c|c|c|c|}
\hline $\begin{array}{l}\text { Outcomes (RR, } \\
95 \% \mathrm{Cl} \text { ) }\end{array}$ & $\begin{array}{l}\text { Excluding } \\
\text { before } \\
2000 \\
\text { trials }^{a}\end{array}$ & $\begin{array}{l}\text { Excluding } \\
\text { open- } \\
\text { label } \\
\text { trials }^{b}\end{array}$ & $\begin{array}{l}\text { Excluding } \\
\text { high risk } \\
\text { trials }^{c}\end{array}$ & $\begin{array}{l}\text { Excluding } \\
\text { asymptomatic } \\
\text { PAD trials }^{\mathrm{d}}\end{array}$ & $\begin{array}{l}\text { Excluding } \\
100 \% \text { male } \\
\text { individual } \\
\text { trials }\end{array}$ & $\begin{array}{l}\text { Excluding } \\
100 \% \text { diabetic } \\
\text { individuals } \\
\text { trials }^{f}\end{array}$ & $\begin{array}{l}\text { Restricting on } \\
100 \% \text { diabetic } \\
\text { individuals } \\
\text { trials }^{g}\end{array}$ & $\begin{array}{l}\text { Excluding } \\
\text { placebo } \\
\text { use } \\
\text { trials }^{h}\end{array}$ & $\begin{array}{l}\text { Excluding } \\
\text { TPT } \\
\text { study }\end{array}$ \\
\hline \multicolumn{10}{|c|}{ Primary efficacy outcomes } \\
\hline CV events & $\begin{array}{l}0.91 \\
(0.87- \\
0.96)\end{array}$ & $\begin{array}{l}0.90 \\
(0.85- \\
0.95)\end{array}$ & $\begin{array}{l}0.91 \\
(0.86- \\
0.97)\end{array}$ & $\begin{array}{l}0.91(0.87- \\
0.96)\end{array}$ & $\begin{array}{l}0.92(0.87- \\
0.97)\end{array}$ & $\begin{array}{l}0.90(0.85- \\
0.95)\end{array}$ & $0.95(0.84-1.06)$ & $\begin{array}{l}0.92 \\
(0.84- \\
1.02)\end{array}$ & NA \\
\hline $\begin{array}{l}\text { All-cause } \\
\text { mortality }\end{array}$ & $\begin{array}{l}0.98 \\
(0.93- \\
1.04)\end{array}$ & $\begin{array}{l}0.98 \\
(0.93- \\
1.03)\end{array}$ & $\begin{array}{l}0.98 \\
(0.92- \\
1.04)\end{array}$ & $\begin{array}{l}0.97(0.93- \\
1.02)\end{array}$ & $\begin{array}{l}0.98(0.93- \\
1.03)\end{array}$ & $\begin{array}{l}0.98(0.93- \\
1.04)\end{array}$ & $0.94(0.86-1.03)$ & $\begin{array}{l}0.93 \\
(0.83- \\
1.03)\end{array}$ & $\begin{array}{l}0.97 \\
(0.93- \\
1.02)\end{array}$ \\
\hline $\begin{array}{l}\text { Cardiovascular } \\
\text { mortality }\end{array}$ & $\begin{array}{l}0.93 \\
(0.82- \\
1.07)\end{array}$ & $\begin{array}{l}0.95 \\
(0.87- \\
1.05)\end{array}$ & $\begin{array}{l}0.95 \\
(0.85- \\
1.05)\end{array}$ & $\begin{array}{l}0.93(0.85- \\
1.02)\end{array}$ & $\begin{array}{l}0.94(0.84- \\
1.04)\end{array}$ & $\begin{array}{l}0.94(0.85- \\
1.05)\end{array}$ & $0.97(0.65-1.45)$ & $\begin{array}{l}0.85 \\
(0.59- \\
1.22)\end{array}$ & $\begin{array}{l}0.95 \\
(0.86- \\
1.03)\end{array}$ \\
\hline \multicolumn{10}{|c|}{ Secondary efficacy outcomes } \\
\hline All MI & $\begin{array}{l}0.95 \\
(0.88- \\
1.03)\end{array}$ & $\begin{array}{l}0.86 \\
(0.74- \\
0.99)\end{array}$ & $\begin{array}{l}0.93 \\
(0.83- \\
1.04)\end{array}$ & $\begin{array}{l}0.84(0.74- \\
0.95)\end{array}$ & $\begin{array}{l}0.92(0.84- \\
1.00)\end{array}$ & $\begin{array}{l}0.83(0.72- \\
0.96)\end{array}$ & $0.97(0.85-1.10)$ & $\begin{array}{l}0.94 \\
(0.79- \\
1.12)\end{array}$ & $\begin{array}{l}0.88 \\
(0.78- \\
0.99)\end{array}$ \\
\hline Total stroke & $\begin{array}{l}0.92 \\
(0.84- \\
1.00)\end{array}$ & $\begin{array}{l}0.94 \\
(0.86- \\
1.03)\end{array}$ & $\begin{array}{l}0.92 \\
(0.84- \\
1.00)\end{array}$ & $\begin{array}{l}0.95(0.89- \\
1.03)\end{array}$ & $\begin{array}{l}0.92(0.85- \\
1.00)\end{array}$ & $\begin{array}{l}0.96(0.88- \\
1.05)\end{array}$ & $0.90(0.88-1.02)$ & $\begin{array}{l}0.98 \\
(0.84- \\
1.15)\end{array}$ & $\begin{array}{l}0.95 \\
(0.88- \\
1.02)\end{array}$ \\
\hline $\begin{array}{l}\text { Ischaemic } \\
\text { stroke }\end{array}$ & $\begin{array}{l}0.86 \\
(0.78- \\
0.94)\end{array}$ & $\begin{array}{l}0.88 \\
(0.78- \\
0.98)\end{array}$ & $\begin{array}{l}0.85 \\
(0.76- \\
0.95)\end{array}$ & $\begin{array}{l}0.88(0.80- \\
0.97)\end{array}$ & $\begin{array}{l}0.86(0.78- \\
0.98)\end{array}$ & $\begin{array}{l}0.86(0.76- \\
0.97)\end{array}$ & $0.92(0.79-1.07)$ & $\begin{array}{l}0.89 \\
(0.72- \\
1.09)\end{array}$ & $\begin{array}{l}0.88 \\
(0.81- \\
0.97)\end{array}$ \\
\hline $\begin{array}{l}\text { Cancer } \\
\text { incidence }\end{array}$ & $\begin{array}{l}1.01 \\
(0.94- \\
1.08)\end{array}$ & $\begin{array}{l}0.99 \\
(0.95- \\
1.05)\end{array}$ & $\begin{array}{l}0.99 \\
(0.95- \\
1.05)\end{array}$ & $\begin{array}{l}1.02(0.97- \\
1.07)\end{array}$ & $\begin{array}{l}1.00(0.94- \\
1.07)\end{array}$ & $\begin{array}{l}1.02(0.95- \\
1.10)\end{array}$ & $0.94(0.82-1.08)$ & $\begin{array}{l}1.06 \\
(0.90- \\
1.25)\end{array}$ & NA \\
\hline $\begin{array}{l}\text { Cancer } \\
\text { mortality }\end{array}$ & $\begin{array}{l}1.03 \\
(0.93- \\
1.15)\end{array}$ & $\begin{array}{l}1.03 \\
(0.92- \\
1.15)\end{array}$ & $\begin{array}{l}1.02 \\
(0.89- \\
1.16)\end{array}$ & $\begin{array}{l}1.05(0.96- \\
1.14)\end{array}$ & $\begin{array}{l}1.03(0.94- \\
1.12)\end{array}$ & $\begin{array}{l}1.04(0.93- \\
1.16)\end{array}$ & $0.98(0.86-1.12)$ & $\begin{array}{l}1,01 \\
(0.86- \\
1.19)\end{array}$ & $\begin{array}{l}1.03 \\
(0.94- \\
1.12)\end{array}$ \\
\hline \multicolumn{10}{|l|}{ Safety outcomes } \\
\hline $\begin{array}{l}\text { Major } \\
\text { bleeding }\end{array}$ & $\begin{array}{l}1.37 \\
(1.12- \\
1.50)\end{array}$ & $\begin{array}{l}1.40 \\
(1.28- \\
1.54)\end{array}$ & $\begin{array}{l}1.39 \\
(1.26- \\
1.53)\end{array}$ & $\begin{array}{l}1.40(1.28- \\
1.52)\end{array}$ & $\begin{array}{l}1.40(1.28- \\
1.54)\end{array}$ & $\begin{array}{l}1.48(1.33- \\
1.64)\end{array}$ & $1.27(1.11-1.47)$ & $\begin{array}{l}1.42 \\
(1.11- \\
1.80)\end{array}$ & $\begin{array}{l}1.40 \\
(1.29- \\
1.52)\end{array}$ \\
\hline $\begin{array}{l}\text { Intracranial } \\
\text { bleeding }\end{array}$ & $\begin{array}{l}1.30 \\
(1.10- \\
1.54)\end{array}$ & $\begin{array}{l}1.33 \\
(1.11- \\
1.59)\end{array}$ & $\begin{array}{l}1.29 \\
(1.07- \\
1.56)\end{array}$ & $\begin{array}{l}1.29(1.10- \\
1.52)\end{array}$ & $\begin{array}{l}1.28(1.08- \\
1.51)\end{array}$ & $\begin{array}{l}1.36(1.14- \\
1.63)\end{array}$ & $1.11(0.80-1.54)$ & $\begin{array}{l}1.22 \\
(0.89- \\
1.68)\end{array}$ & $\begin{array}{l}1.30 \\
(1.11- \\
1.52)\end{array}$ \\
\hline $\begin{array}{l}\text { Gastrointestinal } \\
\text { bleeding }\end{array}$ & $\begin{array}{l}1.49 \\
(1.30- \\
1.72)\end{array}$ & $\begin{array}{l}1.52 \\
(1.33- \\
1.74)\end{array}$ & $\begin{array}{l}1.51 \\
(1.38- \\
1.78)\end{array}$ & $\begin{array}{l}1.58(1.39- \\
1.80)\end{array}$ & $\begin{array}{l}1.55(1.36- \\
1.77)\end{array}$ & $\begin{array}{l}1.63(1.41- \\
1.90)\end{array}$ & $1.43(1.13-1.80)$ & $\begin{array}{l}2.23 \\
(1.33- \\
3.74)\end{array}$ & $\begin{array}{l}1.56 \\
(1.38- \\
1.78)\end{array}$ \\
\hline
\end{tabular}

Note: Sensitivity analysis was conducted by omitting one/several study/studies each turn to show more clinical useful data.

Abbreviations: MI: myocardial infraction; PAD: peripheral artery disease; NA: Not available; RR: Relative risk; Cl: Confidence interval.

a Total 10 trials $^{16-25}, \mathrm{~N}=115300$;

b Total 9 trials ${ }^{13-15}, 17,18,20,23-25, \mathrm{~N}=135042$;

${ }^{c}$ Total 7 trials ${ }^{15,17,18,20,23-25}, \mathrm{~N}=110432$

d Total 12 trials $^{12-17,19,21-25} ; \mathrm{N}=159214$;

e Total 11 trials $^{15-25}, \mathrm{~N}=134090$;

${ }^{f}$ Total 10 trials ${ }^{12-17,20,21,24,25}, \mathrm{~N}=142385$;

g Total 4 trials ${ }^{18,19,22,23}, \mathrm{~N}=21455$;

h Total 5 trials ${ }^{12,16,19,21,22}, \mathrm{~N}=28797$;

i Total 13 trials ${ }^{12,13,15-25}, \mathrm{~N}=161300$. 


\section{Trial sequential analysis}

In TSA, we observed the Z-curve cross the trial sequential analysis boundary (TSA boundary) for CV events, all MI, ischaemic stroke, major bleeding, intracranial bleeding and major gastrointestinal bleeding outcomes under conditions of $5 \%$ relative risk reduction, $5 \%$ for two-sided type 1 error risk, $80 \%$ statistical power and $5 \%$ control event incidence. The Z-curve did not cross the traditional boundary or the TSA boundary but crossed the futility boundary for cardiovascular mortality. The Z-curve crossed the traditional and futility boundaries but did not cross the TSA boundary for all-cause mortality. These findings showed that conclusions on the abovementioned outcomes were robust and were hardly modified with additional related trials. However, the Z-curve did not cross the TSA boundary or the futility boundary for total stroke, cancer incidence and cancer mortality, which suggested that additional studies should be conducted to evaluate those effects (Figure 3 and Appendix Figure S4).

Egger's test revealed no significant publication bias for CV events $(P=0.882)$, all-cause mortality $(P=0.362), C V$ mortality $(P=0.390)$, major bleeding $(P=$ 0.126), intracranial bleeding $(P=0.236)$, or major gastrointestinal bleeding $(P=0.152)$ (Appendix Figure S5).

\section{Discussion}

As one of the most widely used drugs worldwide, aspirin celebrated its 121 st birthday in 2020 and the remarkable store is still going on ${ }^{34}$. In this study, aspirin was observed to be significantly associated with a $9 \%, 13 \%$, and $12 \%$ reduction in the risk of CV events, all-MI and ischemic stroke, respectively; however, aspirin was associated with a $40 \%, 30 \%$, and $57 \%$ increase in the risk of bleeding profiles, including major bleeding, intracranial bleeding and major gastrointestinal bleeding, respectively. No causal outcomes were found in all-cause mortality, cardiovascular mortality, total stroke, cancer incidence or cancer mortality. Low doses of aspirin ( $\leq 100 \mathrm{mg}$ ) might offer more clinical benefits than high doses of aspirin; individuals who are $<65 \mathrm{y}$ old and have a BMI $\geq 25$ demonstrated stronger effects of aspirin on the primary prevention of CVD; the data indicated that aspirin did not confer benefits in the high 10-y MACE\% risk group. The results were not significantly modified after excluding asymptomatic PAD trials and trials with only diabetic individuals. Besides recommendations from contemporary guidelines, we hypothesized that aspirin might be prescribed depending on body size (BMI), that is, prescribing > 100 or $\leq 100$ mg aspirin to individuals with a $\mathrm{BMI} \geqq 25$ and prescribing $\leq 100 \mathrm{mg}$ to individuals with a $\mathrm{BMI}<25^{35}$. It is still crucial to perform complete screening and examinations on large populations to evaluate populations' CVD risk, hence quantifying their probability of obtaining real benefits from aspirin. Indeed, the one-dose-fits-all intake strategy is unlikely optimal, and a more tailored and wise dosing approach is called for to maximize substantial benefits and reduce potential risk.

The endorsed role of aspirin in the primary prevention of ischaemic events (all-MI, ischaemic stroke) has been supported by several studies ${ }^{36}$. The potential mechanism for preventing ischaemic events is based on the inhibition of thrombus propagation and plaque rupture ${ }^{37}$. This study also suggested a beneficial role of aspirin in all-MI and ischaemic stroke outcomes. Notably, only 2 eligible trials (HOT and PHS) ${ }^{13,15}$ exhibited significant risk reduction in all-Ml; however, their conducting time was rather early, and no significant risk reduction was observed in cardiovascular mortality and all-cause mortality under the long followup period. Because the two trials were conducted early, researchers could not properly emphasize the biases from risk factors such as smoking status, blood glucose, blood cholesterol level or blood pressure. Another concern is that almost $50 \%$ of Mls are considered to be clinically silent; accordingly, it is not easy to ascertain the clinical benefit from long-term aspirin use through this endpoint ${ }^{38}$. It may be that all CV events are assessed to be proper endpoints to evaluate all these cases. Some studies have suggested that populations with substantially increased CVD risk may benefit from preventive aspirin use, and guidelines from the US Preventive Services Task Force also suggested prescribing low doses of aspirin in adults aged $50-59$ years with a CVD risk of at least $10 \% 39$, which was in contrast to our findings that low-risk individuals seemed to obtain more clinical benefits. We used the 10-y MACE\% to reflect participants' CVD risk and hypothesized that the CVD risk of participants tended to be overestimated due to the lack of agreement on unified risk calculators in primary trials ${ }^{40}$. For example, the ARRIVE trial ${ }^{24}$ mixed predicted and observed CVD risk, such that the enrolled moderate risk populations had a standard risk of $17.3 \%$ as estimated by American Heart Association (AHA)/American College of Cardiology (ACC) 10-y CV risk estimated criteria ${ }^{40,41}$ but had an observed CVD risk rate of $6.9 \%$. Similarly, the ASPREE trial ${ }^{25}$ enrolled patients who were older than 65 or $70 \mathrm{y}$ old; the CVD risk of these older patients was hard to evaluate, and the reported 10 -y MACE\% of $7.8 \%$ differed from the $8.3 \%$ figure found herein, although both 10 -y MACE\% were less than $10 \%$. The reason for this discrepancy was that MACE in the ASPREE trial was defined as a composite of fatal coronary heart disease, nonfatal MI and fatal or nonfatal ischaemic stroke, which differed from the unified definition. In this study, CV event risk was reduced by $11 \%$ in the low 10 -y MACE\% risk group.

Guidelines driven by the AHA/American Diabetes Association (ADA) recommend aspirin use in diabetic populations with intermediate risk (5\%-10\% 10-y MACE\%) for primary prevention ${ }^{36}$. JPAD ${ }^{19}$ and ASCEND ${ }^{23}$ trials specifically incorporated diabetic populations, but the cardiovascular benefits seemed to be higher in the ASCEND trial. The total proportion of statin use was $75 \%$ in the ASCEND trial vs. $25 \%$ in the JPAD trial, which might have resulted in higher benefits seen in the ASCEND trial. Additionally, this study indicated fewer CVD benefits among populations with diabetes, which was supported by recent European Society of Cardiology guidelines recommending against aspirin use in diabetic populations who have no history of CVD ${ }^{9}$. Routine aspirin use was not enough for primary prevention among individuals with a high risk of CVD; at that time, blood pressure and blood glucose were controlled, cholesterol levels were reduced with statins, and physical activity and healthy eating were reduced. are also necessary. Aspirin use increased the risk of bleeding profiles but was not associated with cardiovascular mortality considering that deaths caused by bleeding were rare. Since the strategy to reduce harm of long-term aspirin use is not understood from current evidence, prescribing proton pump inhibitors (PPIs) might limit the risk of major gastrointestinal bleeding and enhance the benefit-risk ratio towards intended populations ${ }^{20}$. Aspirin appears to be not associated with all-cause mortality; however, several trials revealed that aspirin reduced the risk of colorectal cancer (RR: $0.73,95 \% \mathrm{Cl}$ : 0.69-0.78), squamous-cell oesophageal cancer (RR: 0.67, 95\% Cl: 0.57-0.79), gastric cancer (RR: 0.64, $95 \% \mathrm{Cl}: 0.51-0.82$ ) and pancreatic cancer (RR: $0.78,95 \% \mathrm{Cl}: 0.68-0.89)^{42}$. At this time, the reduction in cancer mortality appeared after 5 y of follow-up, and this result was not duplicated in the ASCEND trial ${ }^{23}$. Current findings suggest a neutral role of aspirin in cancer outcomes; therefore, no suggestions could be made regarding benefit-risk balance from current evidence. 


\section{Added Value And Limitations}

Mahmoud et al ${ }^{43}$ conducted a TSA meta-analysis, the authors mainly focused on CVD-related outcomes including all-cause mortality, all MI, bleeding events. Comparing to Mahmoud et $\mathrm{al}^{43}$, current study is more comprehensive because we also investigated cancer outcomes. Study from Mahmoud et al ${ }^{43}$ included 11 RCTs, in our prospective, it was not enough, trials like POPADAD ${ }^{18}, A A A^{20}$ were not reasonably included. Also, several $10 y-M A C E \%$ values presented in that study were not in consistent with current study, for example ASCEND ${ }^{23}$, ARRIVE ${ }^{24}$ and ASPREE ${ }^{25}$. 10y-MACE\% for BDS ${ }^{12}$ and TPT ${ }^{14}$ was also absent in Mahmoud et $\mathrm{al}^{43}$ study. Lin et $\mathrm{al}^{44}$ investigated the role of low-dose of aspirin on CVD primary prevention, they demonstrated low-dose aspirin had no role in all MI, but did reduce stroke incidence, which was in contrast to findings from current paper (that aspirin might significantly reduce all MI incidence instead of total stroke, ischemic stroke could be reasonably reduced). Current study had included more comprehensive RCTs than Lin et al ${ }^{44}$, subgroup analyses aiming to low-dose of aspirin ( $<100 \mathrm{mg} / \mathrm{d}$ ) were also conducted. This study clearly pinpointed low CVD risk individuals might get more clinical benefits than the high risk from aspirin. Only one TSA for MACE outcome in Lin et $\mathrm{al}^{44}$ was far enough to draw robust conclusions. Major controversial issues from current study and Gelbenegger et al ${ }^{45}$ were the outcomes on diabetic populations, this study supported there were no substantial benefits of aspirin on diabetic populations primary prevention. POPADAD ${ }^{18}, \mathrm{JPAD}^{19}, \mathrm{JPAD}^{22}$ and $\mathrm{ASCEND}^{23}$ were special trials conducted on full diabetic populations (100\% diabetic individuals), to our great knowledge, it was more proper to investigate the intended results on the four trials, data stem from calculation on other small diabetic-proportion trials ${ }^{17}$, ${ }^{21}$ would add extra reporting bias. Zheng et al ${ }^{27}$ also performed a similar research, however, no TSA results were revealed and merits from network metaanalysis methods seemed not so obvious. Overall, current study with particular subgroup and sensitivity analyses clearly addressed the less priority of aspirin on high 10y-MACE\% risk and diabetic populations, such populations may need more aggressive therapy or combined pharmaceutical intervention. We believe these results add new evidence to the discussion on aspirin primary prevention in CVD and may arouse new disputes.

Limitations were also detected. First, definitions of reported outcomes were different, reflecting advances in CVD diagnosis and treatment. To best overcome this heterogeneity, we defined unified primary and secondary efficacy outcomes and safety profiles and then properly extracted the required data in eligible studies. Second, aspirin use in the included studies was not consistent with the major dose of $75 \mathrm{mg}$ to $100 \mathrm{mg}$. Importantly, more clinical benefits with bleeding risk were found in trials restricted to $\leq 100 \mathrm{mg} / \mathrm{d}$ intake. Third, several trials (BDS (1998), PHS (1989), TPT (1998), HOT (1998)) were published rather early, and thus, some examinations and screening methods may not have been as accurate as expected. This contributed to an overestimated 10-y MACE\%. A more precise study based on individual-patient data is encouraged.

\section{Conclusion}

Aspirin intake was associated with reduced risk of CV events, all MI, and ischaemic stroke, and was associated with increased incidences of major bleeding, intracranial bleeding, and major gastrointestinal bleeding in the primary prevention of CVD. The use was not associated with an increased risk of all-cause mortality, cardiovascular mortality, total stroke, cancer incidence or cancer mortality. No substantial benefits with respect to CVD were observed in the diabetic and high 10-y MACE\% risk group populations. A one-dose-fits-all strategy is not optimal, and BMI may be a potential indicator to guide aspirin prescription. It is also necessary to identify individuals who may benefit from aspirin by more accurate cardiovascular-relating examinations. Overall, the benefits and harm of aspirin for primary prevention should be re-evaluated. Based on these findings, we believe it is not yet the time to quit the aspirin era.

\section{Abbreviations}

\section{CVD}

cardiovascular disease; TSA:Trial sequential analysis; CV:events cardiovascular events; RR:relative risk; Cl:confidence intervals; BMl:body mass index; CHD:coronary heart disease; MI:myocardial infarction; PRISMA:Preferred Reporting Items for Systematic Reviews and Meta-Analysis; RCTs:randomized controlled trials; HR:hazard ratio; OR:odds ratio; MACE:major adverse cardiovascular event; Crls:credible intervals; D-L:DerSimonian-Laird; Ess:effect sizes; IV:inverse variance; PAD:peripheral artery disease; AHA:American Heart Association; ACC:American College of Cardiology; ADA:American Diabetes Association.

\section{Declarations}

\section{Ethics approval and consent to participate}

Not applicable.

\section{Consent for publication}

Not applicable.

\section{Availability of data and materials}

All data generated or analyzed during this study are included in this published article and its Additional information fles.

\section{Competing interests}


The authors have completed and submitted the ICMJE Form for Disclosure of Potential Conflicts of Interest. The authors declare that they have no competing interests.

\section{Funding}

This study was supported by National Natural Science Foundation of China (NSFC), with no commercial entity involved, number of grants (81560345).

\section{Authors' contributions}

All authors designed and conducted this review. Binghao Zhao wrote the paper. Qian Wu, Li Wang, Chen Liao, Yifei Dong, Jingsong Xu, Yiping Wei and Wenxiong Zhang helped the study design. Binghao Zhao, Yiping Wei and Wenxiong Zhang revised the statistical methodology. Binghao Zhao and Wenxiong Zhang had primary responsibility for the final content. All authors read and approved the final manuscript. Notably, Binghao Zhao and Wenxiong Zhang equally share the corresponding authorship.

\section{Acknowledgements}

The authors thank Wenbin Ma, MD, PhD (Departments of Neurosurgery, Peking Union Medical College Hospital, Chinese Academy of Medical Sciences and Peking Union Medical College), for his writing instructions and statistical guidance.

\section{References}

1. Listed N. Collaborative overview of randomised trials of antiplatelet therapy-III: Reduction in venous thrombosis and pulmonary embolism by antiplatelet prophylaxis among surgical and medical patients. Antiplatelet Trialists' Collaboration BMJ. 1994;308(6923):235.

2. Antithrombotic Trialists' Collaboration. Collaborative meta-analysis of randomised trials of antiplatelet therapy for prevention of death, myocardial infarction, and stroke in high risk patients. BMJ. 2002;324(7329):71-86.

3. Bibbins-Domingo K. Aspirin Use for the Primary Prevention of Cardiovascular Disease and Colorectal Cancer: U.S. Preventive Services Task Force Recommendation Statement. Ann Intern Med. 2016;164(12):836-45.

4. Pearson TA, Blair SN, Daniels SR, Eckel RH, Fair JM, Fortmann SP, et al. AHA Guidelines for Primary Prevention of Cardiovascular Disease and Stroke: 2002 Update: Consensus Panel Guide to Comprehensive Risk Reduction for Adult Patients Without Coronary or Other Atherosclerotic Vascular Diseases. American Heart Association Science Advisory and Coordinating Committee. Circulation. 2002;106(3):388-391.

5. Elwood P, Morgan G, Brown G, Pickering J. Aspirin for everyone older than 50? For. BMJ. 2005;330(7505):1440-1.

6. Bulugahapitiya U, Siyambalapitiya S, Sithole J, Fernando DJ, Idris I. Age threshold for vascular prophylaxis by aspirin in patients without diabetes. Heart. 2008;94(11):1429-32.

7. Wald NJ, Law MR. A strategy to reduce cardiovascular disease by more than $80 \%$. BMJ. 2003;326(7404):1419.

8. ehaa150

Mortensen MB, Nordestgaard BG. 2019 vs. 2016 ESC/EAS statin guidelines for primary prevention of atherosclerotic cardiovascular disease. Eur Heart J. 2020;ehaa150. doi: 10.1093/eurheartj/ehaa150.

9. Piepoli MF, Hoes AW, Agewall S, Albus C, Brotons C, Catapano AL, et al. 2016 European Guidelines on cardiovascular disease prevention in clinical practice: The Sixth Joint Task Force of the European Society of Cardiology and Other Societies on Cardiovascular Disease Prevention in Clinical Practice (constituted by representatives of 10 societies and by invited experts)Developed with the special contribution of the European Association for Cardiovascular Prevention \& Rehabilitation (EACPR). Eur Heart J. 2016;37(29):2315-81.

10. Grundy SM, Stone NJ, Bailey AL, Beam C, Birtcher KK, Blumenthal RS, et al 2018 AHA/ACC/AACVPR/AAPA/ABC/ACPM/ADA/AGS/APhA/ASPC/NLA/PCNA Guideline on the Management of Blood Cholesterol: A Report of the American College of Cardiology/American Heart Association Task Force on Clinical Practice Guidelines. Circulation. 2019;139(25):e1082-e1143.

11. Fox CS, Golden SH, Anderson C, Bray GA, Burke LE, de Boer IH, et al. Update on Prevention of Cardiovascular Disease in Adults With Type 2 Diabetes Mellitus in Light of Recent Evidence: A Scientific Statement From the American Heart Association and the American Diabetes Association. Diabetes Care. 2015;38(9):1777-803.

12. Peto R, Gray R, Collins R, Wheatley K, Hennekens C, Jamrozik K, et al. Randomised trial of prophylactic daily aspirin in British male doctors. BMJ. 1988;296(6618):313-6.

13. Steering Committee of the Physicians' Health Study Research Group. Final Report on the Aspirin Component of the Ongoing Physicians' Health Study. N Engl J Med. 1989;321(3):129-35.

14. The Medical Research Council's General Practice Research Framework. Thrombosis prevention trial: randomised trial of low-intensity oral anticoagulation with warfarin and low-dose aspirin in the primary prevention of ischaemic heart disease in men at increased risk. Lancet. 1998;351(9098):233-41.

15. Hansson L, Zanchetti A, Carruthers SG, Dahlof B, Elmfeldt D, Julius S, et al. Effects of intensive blood-pressure lowering and low-dose aspirin in patients with hypertension: principal results of the Hypertension Optimal Treatment (HOT) randomised trial. HOT Study Group Lancet. 1998;351(9118):1755-62.

16. de Gaetano G. Low-dose aspirin and vitamin E in people at cardiovascular risk: a randomised trial in general practice. Collaborative Group of the Primary Prevention Project. Lancet. 2001;357(9250):89-95. 
17. Ridker PM, Cook NR, Lee IM, Gordon D, Gaziano JM, Manson JE, et al. A randomized trial of low-dose aspirin in the primary prevention of cardiovascular disease in women. N Engl J Med. 2005;352(13):1293-304.

18. Belch J, MacCuish A, Campbell I, Cobbe S, Taylor R, Prescott R, et al. The prevention of progression of arterial disease and diabetes (POPADAD) trial: factorial randomised placebo controlled trial of aspirin and antioxidants in patients with diabetes and asymptomatic peripheral arterial disease. BMJ. 2008;337:a1840.

19. Ogawa H, Nakayama M, Morimoto T, Uemura S, Kanauchi M, Doi N, et al. Low-dose aspirin for primary prevention of atherosclerotic events in patients with type 2 diabetes: a randomized controlled trial. JAMA. 2008;300(18):2134-41.

20. Fowkes FG, Price JF, Stewart MC, Butcher I, Leng GC, Pell AC, et al. Aspirin for prevention of cardiovascular events in a general population screened for a low ankle brachial index: a randomized controlled trial. JAMA. 2010;303(9):841-8.

21. Ikeda Y, Shimada K, Teramoto T, Uchiyama S, Yamazaki T, Oikawa S, et al. Low-dose aspirin for primary prevention of cardiovascular events in Japanese patients 60 years or older with atherosclerotic risk factors: a randomized clinical trial. JAMA. 2014;312(23):2510-20.

22. Saito Y, Okada S, Ogawa H, Soejima H, Sakuma M, Nakayama M, et al. Low-Dose Aspirin for Primary Prevention of Cardiovascular Events in Patients With Type 2 Diabetes Mellitus: 10-Year Follow-Up of a Randomized Controlled Trial. Circulation. 2017;135(7):659-70.

23. Bowman L, Mafham M, Wallendszus K, Stevens W, Buck G, Barton J, et al. Effects of Aspirin for Primary Prevention in Persons with Diabetes Mellitus. N Engl J Med. 2018;379(16):1529-39.

24. Gaziano JM, Brotons C, Coppolecchia R, Cricelli C, Darius H, Gorelick PB, et al. Use of aspirin to reduce risk of initial vascular events in patients at moderate risk of cardiovascular disease (ARRIVE): a randomised, double-blind, placebo-controlled trial. Lancet. 2018;392(10152):1036-46.

25. McNeil JJ, Wolfe R, Woods RL, Tonkin AM, Donnan GA, Nelson MR, et al. Effect of Aspirin on Cardiovascular Events and Bleeding in the Healthy Elderly. N Engl J Med. 2018;379(16):1509-18.

26. Whitlock EP, Burda BU, Williams SB, Guirguis-Blake JM, Evans CV. Bleeding Risks With Aspirin Use for Primary Prevention in Adults: A Systematic Review for the U.S. Preventive Services Task Force. Ann Intern Med. 2016;164(12):826-35.

27. Zheng SL, Roddick AJ. Association of Aspirin Use for Primary Prevention With Cardiovascular Events and Bleeding Events: A Systematic Review and Meta-analysis. JAMA. 2019;321(3):277-87.

28. Higgins JP, Altman DG, Gotzsche PC, Juni P, Moher D, Oxman AD, et al. The Cochrane Collaboration's tool for assessing risk of bias in randomised trials. BMJ. 2011;343:d5928.

29. De Lima Taga MF, Singer JM. Simple linear regression with interval censored dependent and independent variables. Stat Methods Med Res. 2018;27(1):198-207.

30. Higgins JP, Thompson SG, Deeks JJ, Altman DG. Measuring inconsistency in meta-analyses. BMJ. 2003;327(7414):557-60.

31. Egger M, Davey Smith G, Schneider M, Minder C. Bias in meta-analysis detected by a simple, graphical test. BMJ. 1997;315(7109):629-34.

32. Brok J, Thorlund K, Wetterslev J, Gluud C. Apparently conclusive meta-analyses may be inconclusive-Trial sequential analysis adjustment of random error risk due to repetitive testing of accumulating data in apparently conclusive neonatal meta-analyses. Int J Epidemiol. 2009;38(1):287-98.

33. Wetterslev J, Thorlund K, Brok J, Gluud C. Trial sequential analysis may establish when firm evidence is reached in cumulative meta-analysis. J Clin Epidemiol. 2008;61(1):64-75.

34. Vranckx P, Valgimigli M, Jüni P, Hamm C, Steg PG, Heg D, et al. Ticagrelor plus aspirin for 1 month, followed by ticagrelor monotherapy for 23 months aspirin plus clopidogrel or ticagrelor for 12 months, followed by aspirin monotherapy for 12 months after implantation of a drug-eluting stent: a multicentre, open-label, randomised superiority trial. Lancet. 2018;392(10151):940-9.

35. Rothwell PM, Cook NR, Gaziano JM, Price JF, Belch JFF, Roncaglioni MC, et al. Effects of aspirin on risks of vascular events and cancer according to bodyweight and dose: analysis of individual patient data from randomised trials. Lancet. 2018;392(10145):387-99.

36. Fox CS, Golden SH, Anderson C, Bray GA, Burke LE, de Boer IH, et al. Update on Prevention of Cardiovascular Disease in Adults With Type 2 Diabetes Mellitus in Light of Recent Evidence: A Scientific Statement From the American Heart Association and the American Diabetes Association. Circulation. 2015;132(8):691-718.

37. Cleland JG. Is aspirin useful in primary prevention? Eur Heart J. 2013;34(44):3412-8.

38. Zhang ZM, Rautaharju PM, Prineas RJ, Rodriguez CJ, Loehr L, Rosamond WD, et al. Race and Sex Differences in the Incidence and Prognostic Significance of Silent Myocardial Infarction in the Atherosclerosis Risk in Communities (ARIC) Study. Circulation. 2016;133(22):2141-8.

39. Guirguis-Blake JM, Evans CV, Senger CA, O'Connor EA, Whitlock EP. Aspirin for the Primary Prevention of Cardiovascular Events: A Systematic Evidence Review for the U.S. Preventive Services Task Force. Ann Intern Med. 2016;164(12):804-13.

40. Rana JS, Tabada GH, Solomon MD, Lo JC, Jaffe MG, Sung SH, et al. Accuracy of the Atherosclerotic Cardiovascular Risk Equation in a Large Contemporary, Multiethnic Population. J Am Coll Cardiol. 2016;67(18):2118-30.

41. Allan GM, Nouri F, Korownyk C, Kolber MR, Vandermeer B, McCormack J. Agreement among cardiovascular disease risk calculators. Circulation. 2013;127(19):1948-56.

42. Bosetti C, Santucci C, Gallus S, Martinetti M, La Vecchia C. Aspirin and the risk of colorectal and other digestive tract cancers: an updated meta-analysis through 2019. Ann Oncol. 2020;31(5):558-68.

43. Mahmoud AN, Gad MM, Elgendy AY, Elgendy IY, Bavry AA. Efficacy and safety of aspirin for primary prevention of cardiovascular events: a meta-analysis and trial sequential analysis of randomized controlled trials. Eur Heart J. 2019;40(7):607-17.

44. Lin MH, Lee CH, Lin C, Zou YF, Lu CH, Hsieh CH, et al. Low-Dose Aspirin for the Primary Prevention of Cardiovascular Disease in Diabetic Individuals: A Meta-Analysis of Randomized Control Trials and Trial Sequential Analysis. J Clin Med. 2019;8(5):609.

Page 12/16 
45. Gelbenegger G, Postula M, Pecen L, Halvorsen S, Lesiak M, Schoergenhofer C, et al. Aspirin for primary prevention of cardiovascular disease: a metaanalysis with a particular focus on subgroups. BMC Med. 2019;17(1):198.

\section{Figures}
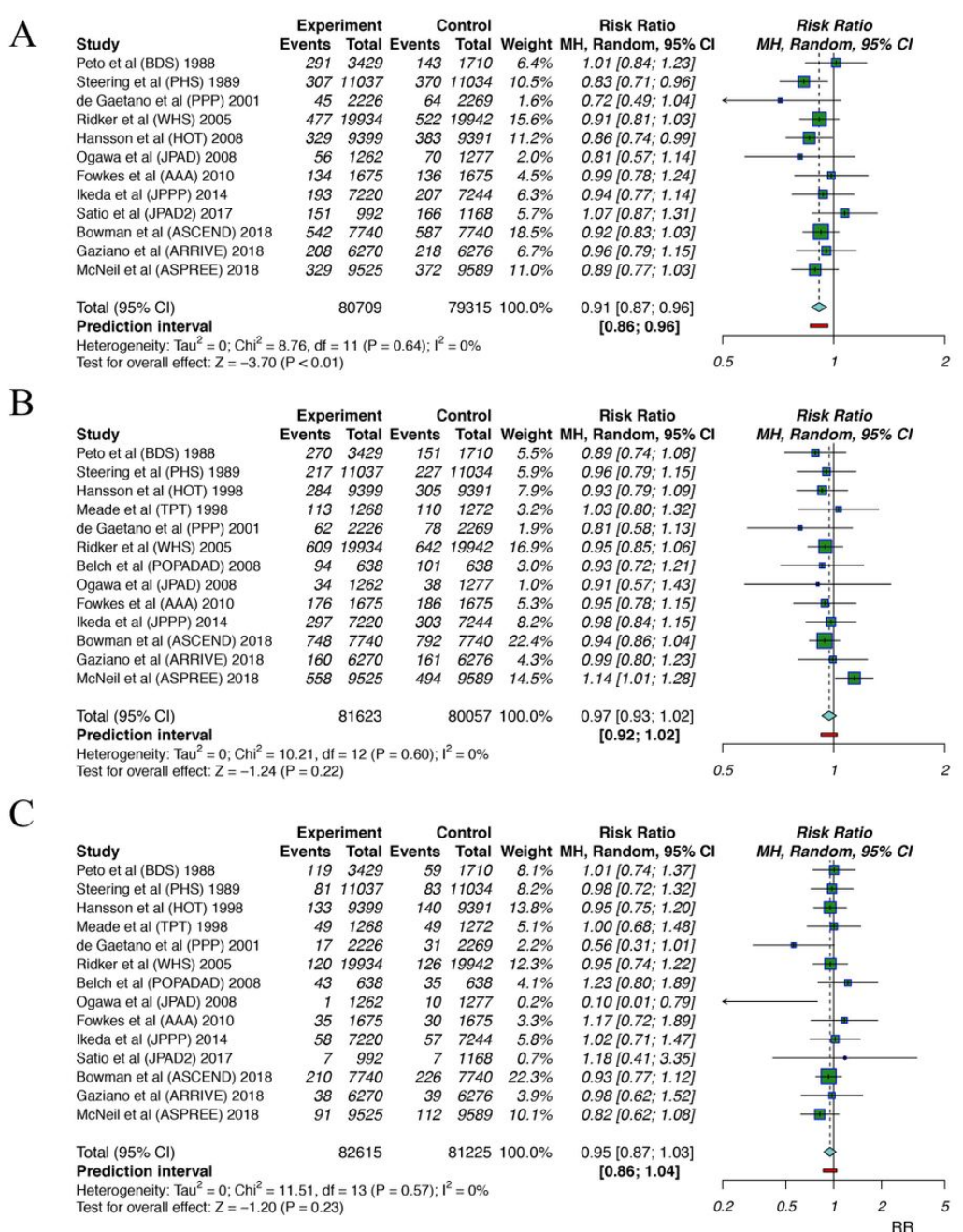

\section{Figure 1}

Summary forest plots for the primary efficacy outcomes. (A) Forest plot for CV events. (B) Forest plot for all-cause mortality. (C) Forest plot for cardiovascular mortality. 
A

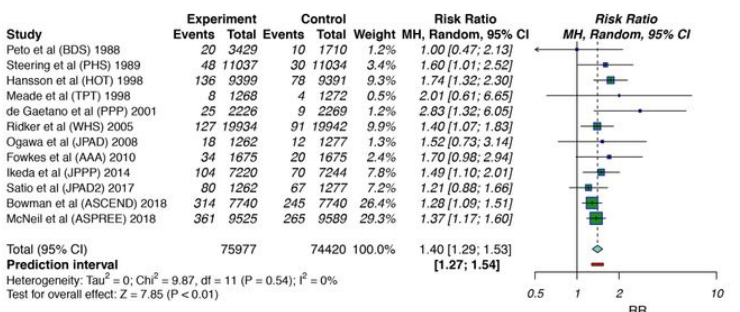

B

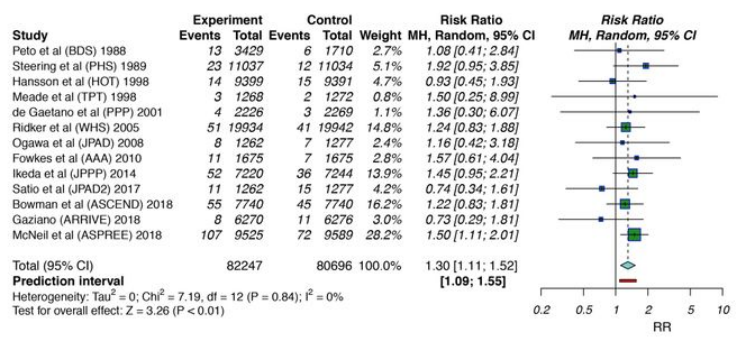

C

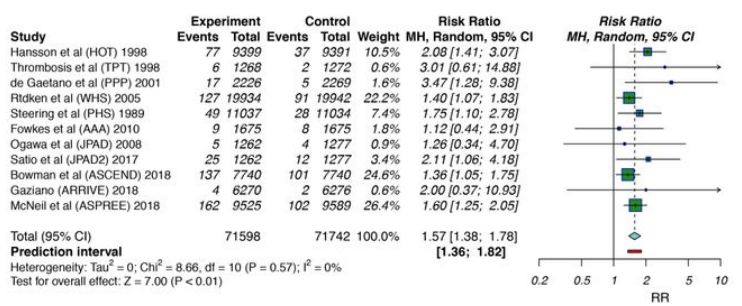

D

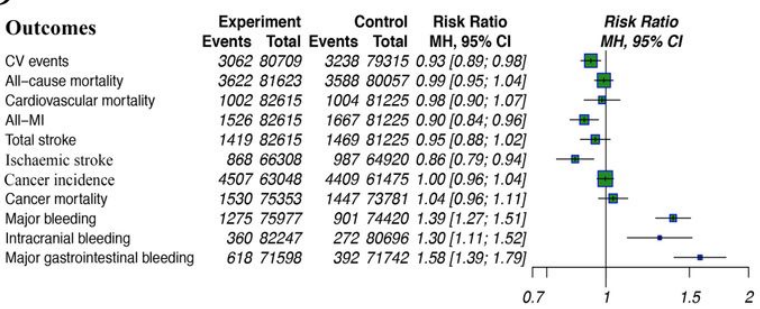

RR

\section{Figure 2}

Summary forest plots for the outcomes of bleeding. (A) Forest plot for major bleeding. (B) Forest plot for intracranial bleeding. (C) Forest plot for major gastrointestinal bleeding. (D) Forest plot for summarized outcomes analysed in the current study. Ml, myocardial infarction; $95 \% \mathrm{Cl}$, 95\% confidence interval. 

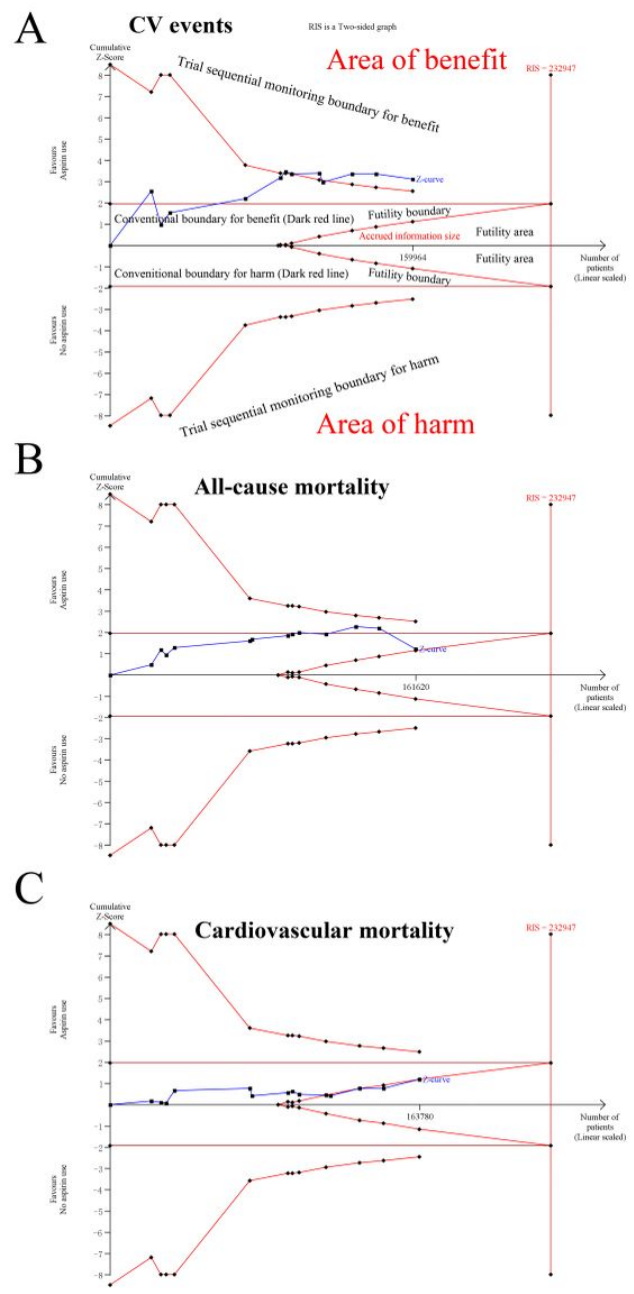

\section{Figure 3}

Trial sequential analysis of CV events, all-cause mortality, and cardiovascular mortality under $5 \%$ relative risk reduction, $5 \%$ for two-sided type 1 error risk, $80 \%$ statistical power and 5\% control event incidence conditions. (A) For CV events. (B) For all-cause mortality. (C) For cardiovascular mortality.

\section{Supplementary Files}

This is a list of supplementary files associated with this preprint. Click to download.

- AppendixFileS12.pdf

- AppendixFileS11.pdf

- AppendixFileS10.pdf

- AppendixFileS9.pdf

- AppendixFiles8.pdf

- AppendixFiles7.pdf

- AppendixFiles6.pdf

- AppendixFileS5.pdf

- AppendixFileS4.pdf

- AppendixFiles3.pdf

- AppendixFiles2.pdf

- AppendixFileS1.docx

- AppendixFigures5.tif

- AppendixFigureS4.tif

- AppendixFigureS3.tif

- AppendixFigures2.tif 
- AppendixFigureS1.tif

- AppendixTables2.docx

- AppendixTableS1.docx 